\title{
Article \\ Efficient Fuzzy Image Stretching for Automatic Ganglion Cyst Extraction Using Fuzzy C-Means Quantization
}

\author{
Sun Joo Lee ${ }^{1}$, Doo Heon Song ${ }^{2}\left(\mathbb{D}\right.$, Kwang Baek Kim ${ }^{3, *} \mathbb{D}$ and Hyun Jun Park ${ }^{4, *(D)}$ \\ 1 Department of Radiology, College of Medicine, Inje University, Busan Paik Hospital, Busan 47392, Korea; \\ sunjulee98@naver.com \\ 2 Department of Computer Games, Yong-in Art and Science University, Yong-in 17145, Korea; \\ dsong@yasu.ac.kr \\ Department of Artificial Intelligence, Silla University, Busan 46958, Korea \\ 4 Division of Software Convergence, Cheongju University, Cheongju 28503, Korea \\ * Correspondence: gbkim@silla.ac.kr (K.B.K.); hyunjun@cju.ac.kr (H.J.P.)
}

Citation: Lee, S.J.; Song, D.H.; Kim, K.B.; Park, H.J. Efficient Fuzzy Image Stretching for Automatic Ganglion Cyst Extraction Using Fuzzy C-Means Quantization. Appl. Sci. 2021, 11, 12094. https://doi.org/ 10.3390/app112412094

Academic Editors: Flavia Ravelli and Francesco Bianconi

Received: 19 October 2021

Accepted: 15 December 2021

Published: 19 December 2021

Publisher's Note: MDPI stays neutral with regard to jurisdictional claims in published maps and institutional affiliations.

Copyright: (c) 2021 by the authors. Licensee MDPI, Basel, Switzerland. This article is an open access article distributed under the terms and conditions of the Creative Commons Attribution (CC BY) license (https:// creativecommons.org/licenses/by/ $4.0 /)$.

\begin{abstract}
Ganglion cysts are commonly observed in association with the joints and tendons of the appendicular skeleton. Ultrasonography is the favored modality used to manage such benign tumors, but it may suffer from operator subjectivity. In the treatment phase, ultrasonography also provides guidance for aspiration and injection, and the information regarding the accurate location of the pedicle of the ganglion. Thus, in this paper, we propose an automatic ganglion cyst extracting method based on fuzzy stretching and fuzzy C-means quantization. The proposed method, with its carefully designed image-enhancement policy, successfully detects ganglion cysts in 86 out of 90 cases (95.6\%) without requiring human intervention.
\end{abstract}

Keywords: ultrasonogram; image enhancement; ganglion cyst; fuzzy stretching; fuzzy C-means

\section{Introduction}

Ganglion cysts are common benign soft tissue tumors that are primarily encountered in the wrist. A history of trauma is elicited in at least $10 \%$ of cases and is considered a causative factor, although the pathogenesis remains unclear [1]. Patients with ganglion cysts may not feel pain, but appropriate treatment is required when the patient feels stiffness or experiences interference with the movement of their joints, and a high recurrence rate is reported even after surgical or non-surgical treatment [2].

Upon examination, the cystic structures of wrist ganglion cysts are usually $1-2 \mathrm{~cm}$ in size [3]. Statistically, more than half of wrist ganglion cysts are found in the dorsal component of the scapholunate ligament, but they can also be found in several other sites across the dorsal aspect of the wrist capsule [1,3]. Microscopically, the pedicle contains a tortuous lumen, connecting the cyst to the underlying joint [4]. Moreover, the presence of a daughter cyst of a preliminary ganglion arising around the joint capsule is often seen. Usually, it can be easily diagnosed by clinical features or location, but it can also be clinically confused with other masses if it is accompanied by complications or when it occurs at an unusual site [5]. The ganglion adjacent to the radial artery near the radiocarpal joint may be pulsatile and that may cause a possible clinical misidentification as a pseudoaneurysm [6].

There are non-surgical and surgical options for treating a ganglion cyst. Nonsurgical treatments of ganglion, including aspiration, steroid injection sclerotherapy, and hyaluronidase, are generally ineffective, although they do have lower complication rates. Open surgical excursions have a lower recurrence rate, but they have higher complication rates and longer recovery periods $[7,8]$. The recurrence rate can be reduced when the pedicle of the ganglion is completely removed during surgery [8]. Therefore, it is helpful to plan surgery to accurately identify the location of the pedicle during imaging.

Ultrasonography is, in general, an effective imaging method for evaluating a palpable soft tissue abnormality, for it has a strong ability to differentiate a solid mass from a cyst. In 
soft tissue lesions, the locations are epidermis, dermis, subcutaneous fat layer, and muscle layer. It is important to recognize the exact location of the ganglion, as well as daughter cysts and the pedicle, before surgery [2].

In the treatment phase, ultrasonography also provides guidance for aspiration and injection [3,9], as well as information regarding the exact location of the pedicle for surgery [8]. The most common reason for ganglion recurrence after surgery is that the pedicle connected to the joint is not completely removed [10].

However, the common complaint about using ultrasonography in diagnosis is its operator subjectivity in that the correctness of sonographic image analysis is largely dependent on the quality of the equipment and the operators' expertise [10]. For example, beginners easily misdiagnose the exact location of the ganglion and overlook the presence of the pedicle.

To avoid such subjectivity, we need an automatic image segmentation and identification tool for anatomical landmarks in the image analysis [11]. It is a difficult problem since the input image may not have sufficient contrast between target object and the background or it contains speckle noise, which is an inherent property of ultrasound imaging modality [12].

In this paper, we propose an efficient automatic segmentation method with carefully designed contrast enhancement by fuzzy stretching. Only surgically confirmed ganglion cysts were included in this study. The purpose of this study is to determine the extent of the ganglion cysts, as well as the daughter cysts, and to find the pedicle accurately and automatically within the ultrasound image using an intelligent pixel clustering method.

Unfortunately, there is no directly comparable research in this field other than our previous attempts. Our first pilot study [5] applied fuzzy stretching to assist image enhancement and then a contour tracking and region labelling method carried out the rest of the identification process; however, because it assumed that the shape of the cyst was oval, the accuracy of this study was not satisfactory. Later, we decided to apply the pixel clustering approach that decides the membership of a pixel to a clustered object based on fuzzy logic, so that the automatic cyst-detecting algorithm was not necessarily dependent on the shape's assumption. An approach with Possibilistic C-Means (PCM) appeared to be effective against speckle noise but tended to underestimate the cyst region, especially when candidate objects overlapped [13]. Fuzzy C-Means (FCM) replaced PCM in forming clusters [14] and generally showed better result.

FCM is a popular unsupervised machine learning algorithm that assigns each datum a degree of fuzzy membership, with the distance measured to the nearest cluster centroid [15]. FCM allows each datum (pixel in the image in this case) to belong to two or more clusters with respect to the degree of membership in each cluster. Thus, FCM classifies the image into clusters with similar pixels in the feature space, iteratively minimizing the cost function defined by the distance between the pixel and the candidate cluster centers in the feature domain. With such flexibility, FCM has been successful in solving segmentation problems in many medical and engineering domains [16-22]. However, it still suffers from object disconnection problems during learning, and our retrospective analysis of it [14] concluded that we need a better image-enhancement policy to overcome the difficulty of a cyst forming during the FCM process.

Thus, we propose a better fuzzy stretching algorithm based on the trapezoid type of membership function under the FCM pixel clustering framework. In this experiment, we also investigate the validity of FCM quantization in forming a cyst by comparing it with ART2 learning [23], which was recently successful in extracting soft tissue tumor.

Details of the image-enhancement algorithm are explained in Section 2, while the cyst extraction process by means of FCM is described in Section 3. Experimental result analysis is then discussed in Section 4, followed by a summary of this paper's main contribution in Section 5 . 


\section{Fuzzy Stretching with Trapezoid Membership Function in Image Enhancement}

Fuzzy stretching is performed in the first place to obtain better image enhancement. Image enhancement is a process of converting the visual appearance of the image into a better image compared with the original image. It is usually used as a support for better analysis results $[24,25]$. Furthermore, in many cases of such human organ ultrasonography, the area of the target organ is often too dark, meaning that there can be important information loss after binarization, which may affect later object forming processes. This was the motivation for developing a more contrast-sensitive membership function for this ganglion cyst extraction problem.

The first step is to compute the average brightness value by using this formula:

$$
x_{m}=\sum_{i=0}^{255} x_{i} \frac{1}{M N}
$$

where $M$ and $N$ denote the width and length of the image.

Then, the distances between the brighter area, the darker area and the average area are computed.

$$
\begin{aligned}
& d_{\text {max }}=\left|x_{h}-x_{m}\right| \\
& d_{\text {min }}=\left|x_{m}-x_{l}\right|
\end{aligned}
$$

where $x_{h}$ and $x_{l}$ are the highest and lowest intensity pixel values, respectively.

The brightness value is adjusted using the following rule:

$$
\begin{gathered}
\text { If }\left(x_{m}>128\right) \text { ad }=255-x_{m} \\
\text { else if }\left(x_{m} \leq d_{\text {min }}\right) \text { ad }=d_{\text {min }} \\
\text { else if }\left(x_{m} \leq d_{\text {max }}\right) \text { ad }=d_{\text {max }} \\
\text { else ad }=x_{m} \\
I_{\text {max }}=x_{m}+a d \\
I_{\text {min }}=x_{m}-a d
\end{gathered}
$$

where $I_{\max }$ and $I_{\min }$ are maximum and minimum intensity, respectively.

Then, we designed a trapezoid type of membership function, as shown in Figure 1b. Previously, we used a typical triangle-type function with dynamic control [13], as shown in Figure 1a. However, that stretching algorithm experienced some information loss in the process, so that the cyst object had been underestimated when the background intensity was similar to that of the cyst object. The trapezoid membership function was more robust in cases such as Lumber Scoliosis X-ray and Lipoma Ultrasonography [26]. Thus, the membership degree $(\mu(I))$ is computed as Equation (5) over the interval $\left[I_{\min }, I_{\max }\right]$. In Figure $1 \mathrm{~b}$, the red lines denote overlapped membership functions, which are explained in Figure 2, and the membership degree is qualitatively categorized as one of L(low), $\mathrm{M}$ (middle), and $\mathrm{H}$ (high).

$$
\begin{aligned}
& I_{\text {mid }}=\frac{I_{\text {max }}+I_{\text {min }}}{2} \\
& I_{\text {mid } 1}=\frac{I_{\text {mid }}+I_{\text {min }}}{2} \\
& I_{\text {mid } 2}=\frac{I_{\text {max }}+I_{\text {mid }}}{2}
\end{aligned}
$$

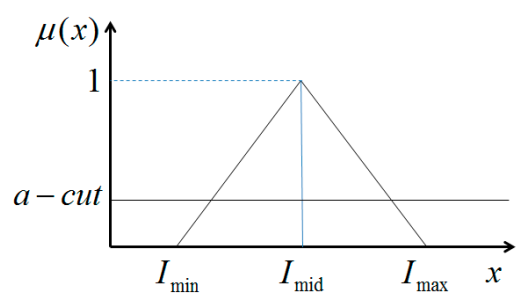

(a)

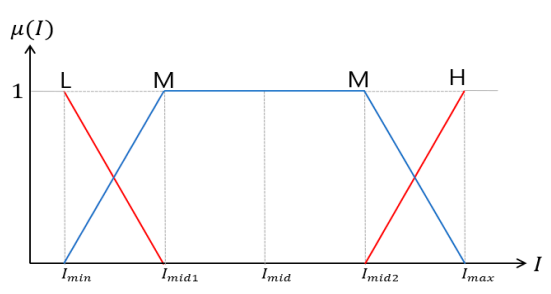

(b)

Figure 1. Fuzzy membership function for fuzzy brightness stretching. (a) Triangle membership function [24], (b) trapezoid membership function (proposed). 


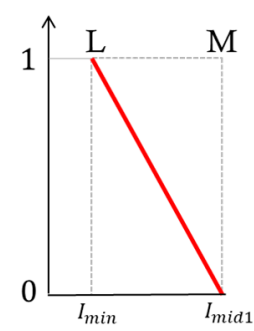

(a)

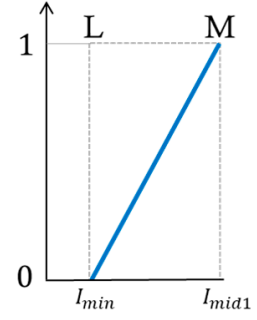

(b)

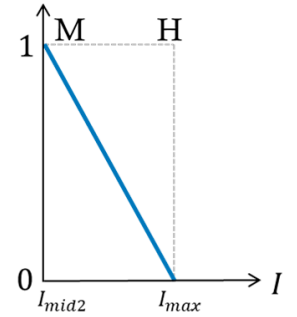

(c)

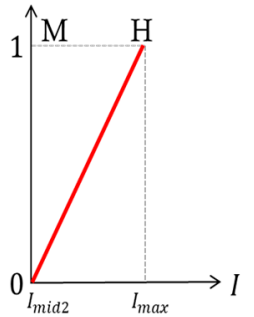

(d)

Figure 2. Fuzzy membership functions for lower and upper limits. (a) Lower limit (dec.), (b) lower limit (inc.), (c) upper limit (dec.), (d) upper limit (inc.).

Previously, the upper limit value $(\beta)$ and the lower limit value $(\alpha)$ are defined as the highest and lowest $X_{i}$ among pixels that have higher membership degrees. However, in this proposed trapezoid membership function, we designed an input membership function as shown in Figure 2 where there are decreasing and increasing lower limits (Figure 2a,b) and upper limits that represent the left and right parts of Figure $1 \mathrm{~b}$.

Figure 3 represents the output function with respect to the membership degree for the lower and upper limits based on the trapezoid structure. In Figure $3, W_{\min }=I_{\min }$ $W_{\text {mid } 1}=I_{\text {mid } 1}, W_{\text {mid } 2}=I_{\text {mid } 2}, W_{\max }=I_{\max }$.

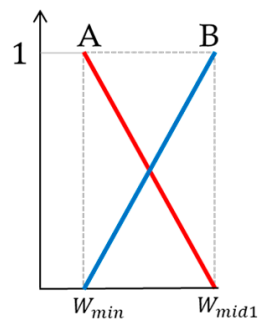

(a)

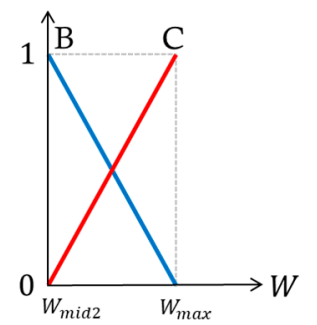

(b)

Figure 3. Fuzzy output membership functions for lower and upper limit. (a) Lower limit, (b) Upper limit.

Then, we made a set of fuzzy inference rules to decide the final stretched result, as shown in Table 1 where I-U and I-U denote the lower and upper limit parts of the membership function value $\mu(I)$. W denotes the output value where its qualitative category is shown in Figure 3.

Table 1. Fuzzy inference rules for fuzzy stretching.

\begin{tabular}{ll}
\hline R1 & If $\mathrm{I}-\mathrm{L}$ is $\mathrm{L}$ and $\mathrm{I}-\mathrm{U}$ is $\mathrm{L}$, then $\mathrm{W}$ is $\mathrm{A}$ \\
\hline R2 & If $\mathrm{I}-\mathrm{L}$ is $\mathrm{L}$ and $\mathrm{I}-\mathrm{U}$ is $\mathrm{M}$, then $\mathrm{W}$ is $\mathrm{A}$ \\
\hline R3 & If $\mathrm{I}-\mathrm{L}$ is $\mathrm{M}$ and $\mathrm{I}-\mathrm{U}$ is $\mathrm{L}$, then $\mathrm{W}$ is $\mathrm{A}$ \\
\hline R4 & If $\mathrm{I}-\mathrm{L}$ is $\mathrm{M}$ and $\mathrm{I}-\mathrm{U}$ is $\mathrm{H}$, then $\mathrm{W}$ is $\mathrm{C}$ \\
\hline R5 & If $\mathrm{I}-\mathrm{L}$ is $\mathrm{H}$ and $\mathrm{I}-\mathrm{U}$ is $\mathrm{M}$, then $\mathrm{W}$ is $\mathrm{C}$ \\
\hline R6 & If $\mathrm{I}-\mathrm{L}$ is $\mathrm{H}$ and $\mathrm{I}-\mathrm{U}$ is $\mathrm{H}$, then $\mathrm{W}$ is $\mathrm{C}$ \\
\hline
\end{tabular}

Then, the final $\alpha$ and $\beta$ values are defuzzified by Equation (6) as follows:

$$
\alpha=\frac{\sum_{i=0}^{\text {mid }} u\left(W_{i}\right) W_{i}}{\sum_{i=0}^{\text {mid }} u\left(W_{i}\right)}, \beta=\frac{\sum_{j=\text { mid }}^{\text {max }} u\left(W_{j}\right) W_{j}}{\sum_{j=\text { mid }}^{\text {max }} u\left(W_{j}\right)}
$$


Then, the final stretched value is given as (7):

$$
f(I)=\frac{I-\alpha}{\beta-\alpha} \times 255
$$

where $f(I)$ denotes the new brightness value.

The effect of this proposed fuzzy stretching is shown in Figure 4.

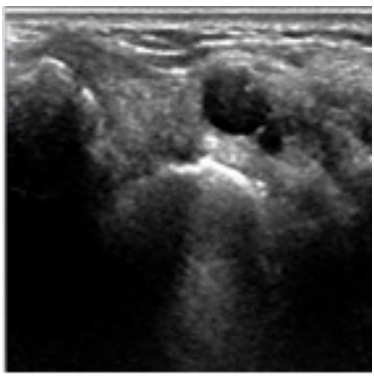

(a)

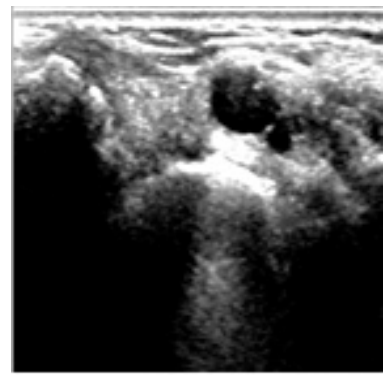

(b)

Figure 4. Effect of fuzzy stretching. (a) Input image, (b) fuzzy stretched.

After stretching, we need to smooth the boundary lines by monotonic cubic spline interpolation [27]. The effect of such smoothing is shown in Figure 5.

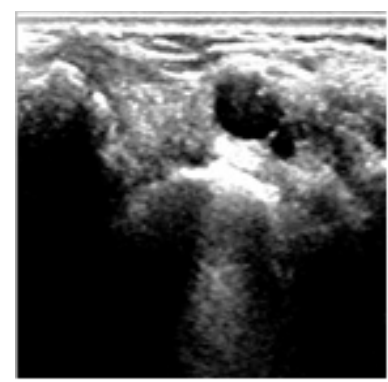

(a)

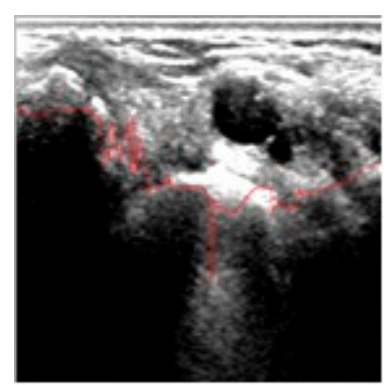

(b)

Figure 5. Effect of cubic spline interpolation. (a) After stretching, (b) after cubic spline.

\section{Cyst Extraction with FCM Algorithm}

The next step is FCM-based quantization [28]. The FCM algorithm is an unsupervised clustering method that has been widely used for ultrasound image analysis whereby pixels with the same features are grouped into the same cluster. The FCM-based quantization algorithm used in this paper is as follows:

Step 1: Initialize the number of cluster $c(2 \leq c<n)$, exponential weight $\mathrm{m}(1 \leq m<\infty)$, the membership degree $u(0)$, and the error threshold $(\varepsilon)$.

Step 2: Compute the central vector $V_{i j}$ as Equation (8) for $\left\{v_{i} \mid i=1,2, \ldots, c\right\}$.

$$
V_{i j}=\frac{\sum_{k=1}^{n}\left(U_{i k}\right)^{m} X_{k j}}{\sum_{k=1}^{n}\left(U_{i k}\right)^{m}}
$$

where $X$ is the input pattern, $i$ is the cluster index, and $j$ is the pattern node index. $k$ is the pattern index, $n$ is the number of patterns, and $U$ is the membership function.

Step 3: Define the FCM cost function $J$ as Equation (9) where $d_{i k}$ is the distance between the $k$-th pattern $x_{k}$ and the central vector of the $i$-th cluster, and $u_{i k}$ is the membership degree of $x_{k}$ among patterns in the $i$-th cluster.

$$
J\left(U_{i k}, v_{i}\right)=\sum_{i=1}^{c} \sum_{k=1}^{n}\left(U_{i k}\right)^{m}\left(d_{i k}\right)^{2}
$$


To minimize $J, d_{i k}$ and membership function $U$ are defined as Equations (10) and (11), respectively.

$$
\begin{gathered}
d_{i k}=\sqrt{\sum_{i=1}^{l}\left(x_{k j}-v_{i j}\right)^{2}} \\
U_{i k}=\frac{1}{\sum_{i=1}^{C}\left(\frac{d_{i k}}{d_{j k}}\right)^{\frac{2}{m-1}}}
\end{gathered}
$$

where $l$ is the number of pattern nodes and $C$ is the number of clusters.

Step 4: Compute the difference between the new and previous membership degrees $\left(U_{i k}\right.$ $\left.(r+1)-U_{i k}(r)\right)$. If the difference is larger than the error threshold $(\varepsilon)$, then go to Step 2; otherwise the algorithm stops.

The effect of FCM-based quantization is shown in Figure 6.

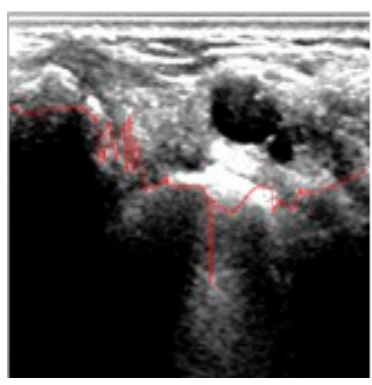

(a)

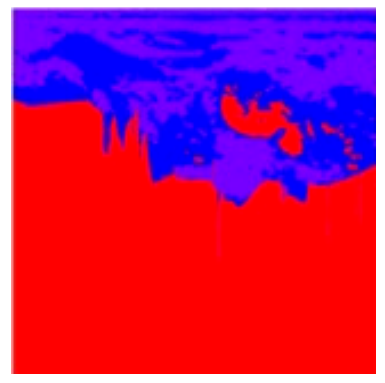

(b)

Figure 6. Effect of FCM-based quantization. (a) Region of interest, (b) FCM quantization.

To remove noises and to clarify the target region effectively, we first apply an "expansion" operation to the image. The "expansion" operator expands the "white pixel" in size so that the bright area can be more emphasized. The brighter area is then expanded by filling adjacent neutral pixels as white and connecting these pixels to form the candidate object. The 8-directional contour tracking algorithm that is explained in detail in [28] is applied to remove such noises using masks and directional search. The object found in the search process that is less than $10 \%$ of the given image or pixels surrounded by zeros are removed as noise. The effect of the 8-directional search is shown in Figure 7.

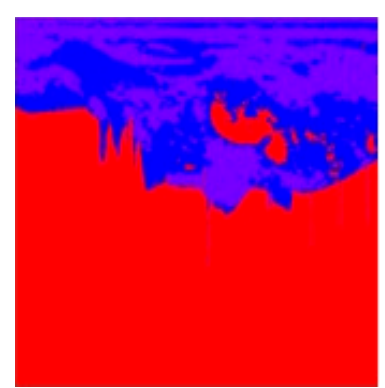

(a)

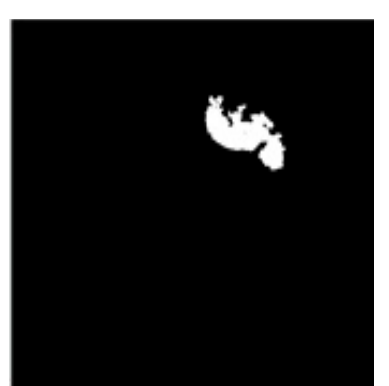

(b)

Figure 7. Effect of 8-directional search. (a) Quantized result, (b) candidate cyst.

Connected component labeling [29] is a simple and efficient algorithm. It is applied after the image has been segmented. We call it a connected component if the pixels have a similar color and are adjacent to each other. Every connected component in the image is labeled uniquely.

The ultrasound image is represented by intensity levels from 0 to 255 in grayscale. From this image, we can differentiate bones, tissues, and fluid. Fluid is dark, tissue is gray, and bone is bright. Because of the segmentation, the ultrasound image, which originally 
has a $0-255$ range of grayscale, turns into an image that has $k$ groups of grayscales $(k=4$ in this paper).

Since the ganglion cyst contains a fluid that is darker in the image, the darkest intensity group is set as foreground color while others are determined as background color. Labeled objects that are too small or too big are removed as noise. After the labeling process is complete, we extract the cyst area by focusing on the labeled object, which is located in the upper center of the image. The extracted cyst image is then colored red, as shown in Figure $8 b$.

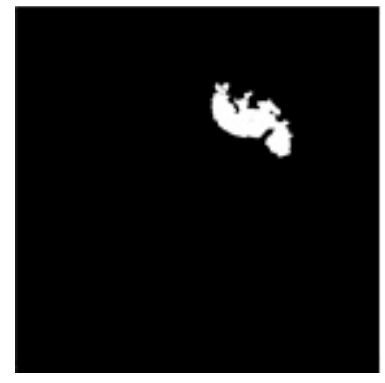

(a)

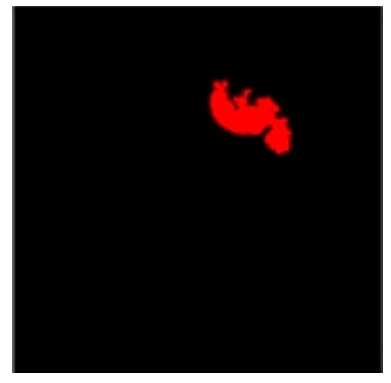

(b)

Figure 8. Ganglion cyst extraction with labelling. (a) Candidate cyst, (b) cyst after labelling.

\section{Results and Discussion}

The experiment is implemented using Visual Studio 2010 C\# with Intel ${ }^{\circledR}$ Core $^{\mathrm{TM}}$ i5 CPU @ 2.80GHz and 8GB RAM with 90 ultrasonography images of wrists containing a ganglion cyst.

Since the articulated point of the proposed method lies in the new fuzzy rule-based stretching, we compare the effect of the proposed stretching with the previous approach used in [24], as shown in Figure 9, to demonstrate its better contrast.

(a)
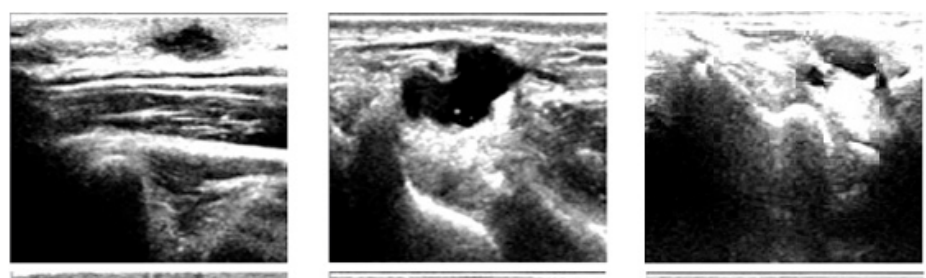

(b)
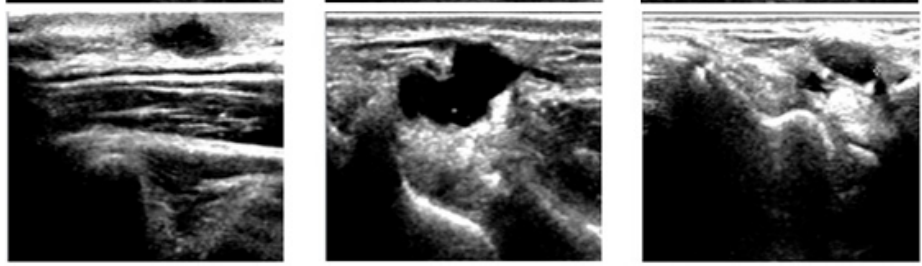

Figure 9. Comparison of fuzzy Stretching. (a) Previous [24]. (b) proposed.

In the quantization process, we used FCM, as demonstrated in Figure 6. However, there is another alternative for intelligent quantization-ART2 learning. ART2 is also an unsupervised real-time stable learning algorithm that does not suffer from the local minima, and it was very successful in addressing the automatic soft tissue extraction problem [24]. We compare the proposed FCM with the ART2 applied in [24], as shown in Figure 10. 
(a)
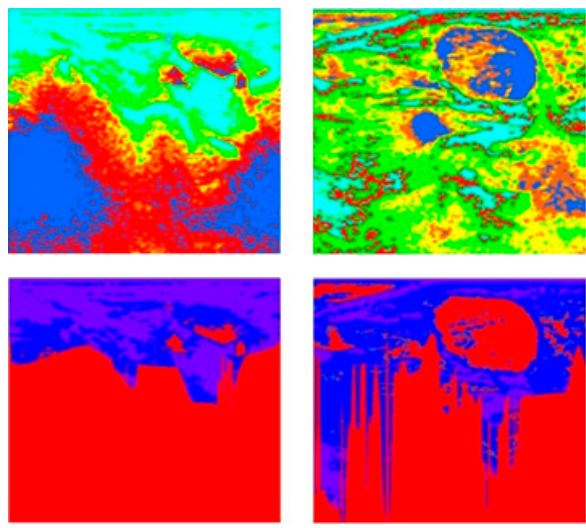
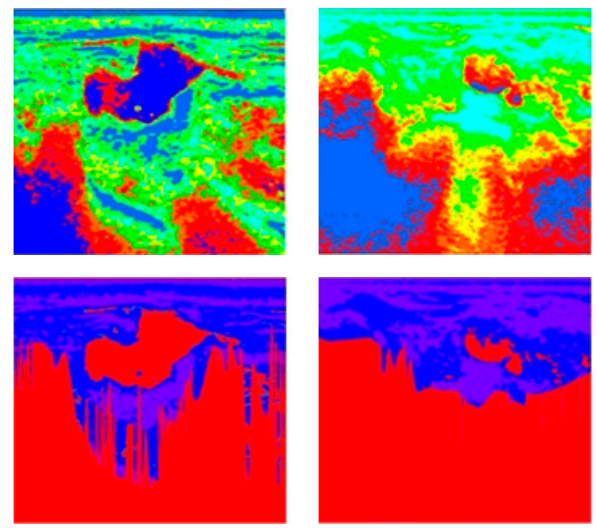

Figure 10. Comparison of quantization: ART2 in [24] vs. proposed FCM. (a) ART2, (b) FCM.

As can be seen from Figure 10, in this specific problem domain, where the purpose of pixel clustering is to stretch the intensity, our proposed FCM quantization (lines of Figure 10b) appears to be more effective than ART2 quantization when used in [24] (lines of Figure 10a) with the same input images. One possible explanation for this is that, in ART2 quantization, the vigilance parameter is defined in a static manner before execution, as shown in Table 2, whereas the proposed FCM takes the cluster based on the fuzzy membership rate, meaning that there are more chances of misclassification in ART2. Moreover, the proposed image-enhancement algorithm makes a more robust decision on pixel classification during the FCM process, whereas fuzzy ART suffers from lowcontrast, irregular-intensity distribution of given input images, as shown in Figure 10. However, ART2 performed better than FCM in other diagnosis problems [30]; therefore, this performance comparison is limited to this specific problem domain.

Table 2. Experiment Parameters.

\begin{tabular}{ccccc}
\hline Method & \multicolumn{2}{c}{ ART2 } & \multicolumn{2}{c}{ FCM } \\
\hline \# of Images & $\begin{array}{c}\text { Vigilance } \\
\text { Parameter }\end{array}$ & \# of Clusters & Weight & $\begin{array}{c}\text { \# of Initial } \\
\text { Clusters }\end{array}$ \\
\hline 90 & 0.1 & 16 & 2 & 10 \\
\hline
\end{tabular}

The accuracy of the automatic extraction of a ganglion cyst in this experiment is summarized in Table 3. The correctness of each cyst extraction is based on the pathologists' agreement over the same input image.

Table 3. Accuracy of Cyst Extraction.

\begin{tabular}{ccc}
\hline Method & ART2 [23] & FCM \\
\hline Correct & 80 & 86 \\
Incorrect & 10 & 4 \\
Accuracy $(\%)$ & 88.9 & 95.6 \\
\hline
\end{tabular}

We show some examples of clearer, more successful extractions of the proposed FCM, as compared with the ART2 method, in Figure 11. 


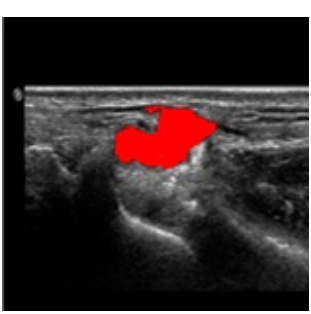

(a)

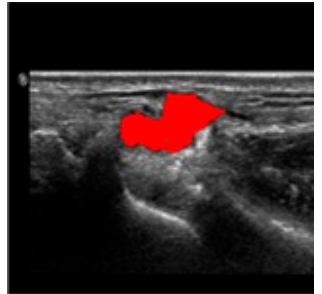

(b)

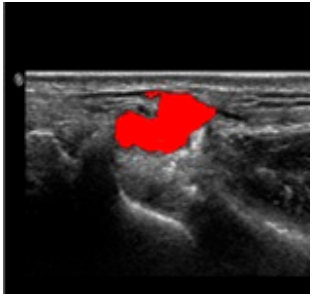

(c)

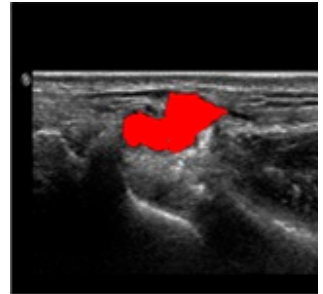

(d)

Figure 11. Comparison of final cyst extractions: ART2 vs. FCM. (a) Case 1 (ART), (b) proposed, (c) Case $2(\mathrm{ART}),(\mathbf{d})$ proposed.

\section{Conclusions}

In this paper, we propose a method to automatically detect ganglion cysts in wrist ultrasonography images using FCM-based quantization with a fuzzy logic-based imageenhancement algorithm. The proposed method shows successful extractions in 86 out of 90 cases (or $95.6 \%$ accuracy), based on the pathologists' evaluation. The power of the proposed method largely lies in our image enhancement policy, which adopts a trapezoidtype membership function and fuzzy inference rules to decide the fuzziness of the intensity stretching. With this automatic ganglion cyst detector software, medical experts can find the cyst area without inspector effect (or operator subjectivity).

In the experiment, the proposed method showed more robust pixel clustering than fuzzy ART, which is another well-known pixel clustering algorithm. The advantage of the proposed method over fuzzy ART is the flexible qualitative fuzzy membership control over low-contrast or irregular-intensity distribution occurs frequently in wrist cyst ultrasonography. The improved fuzzy stretching method used in this paper also mitigates the shape-related sensitive clustering found in previous FCM application of the same domain [14]. However, the proposed method does not consider dynamic control of the number of clusters in FCM process. The main contribution of this paper is to propose a robust automatic ganglion cyst segmentation method to mitigate operator subjectivity of ultrasound image analysis, to make better diagnoses, and to detect the accurate location of cysts.

Author Contributions: Conceptualization, K.B.K. and D.H.S.; methodology, K.B.K. and S.J.L.; software, K.B.K. and H.J.P.; analysis, K.B.K. and D.H.S.; resources, K.B.K. and S.J.L.; data curation, K.B.K. and S.J.L.; writing—original draft preparation, K.B.K. \& H.J.P.; writing—review and editing, K.B.K. and S.J.L.; visualization, D.H.S. and H.J.P.; super-vision, K.B.K. and H.J.P.; project administration, S.J.L.; funding acquisition, S.J.L. All authors have read and agreed to the published version of the manuscript.

Funding: This work was supported by grant from Inje University, 2019.

Institutional Review Board Statement: Not applicable.

Informed Consent Statement: Informed consent was obtained from all subjects involved in the study.

Data Availability Statement: The data presented in this study are available on request from the corresponding author. The data are not publicly available due to Institutional regulations.

Acknowledgments: This work was supported by grant from Inje University, 2019.

Conflicts of Interest: The authors declare no conflict of interest regarding the publication of this paper.

\section{References}

1. Meena, S.; Gupta, A. Dorsal wrist ganglion: Current review of literature. J. Clin. Orthop. Trauma 2014, 5, 59-64. [CrossRef]

2. Freire, V.; Guérini, H.; Campagna, R.; Moutounet, L.; Dumontier, C.; Feydy, A.; Drapé, J.L. Imaging of Hand and Wrist Cysts: A Clinical Approach. Am. J. Roentgenol. 2012, 199, W618-W628. [CrossRef] [PubMed] 
3. Gude, W.; Morelli, V. Ganglion Cysts of the Wrist: Pathophysiology, Clinical Picture, and Management. Curr. Rev. Musculoskelet. Med. 2008, 1, 205-211. [CrossRef] [PubMed]

4. Tophoj, K.; Henriques, U. Ganglion of the wrist-a structure developed from the joint. Acta Orthop. Scand. 1971, 42, 244-250. [CrossRef] [PubMed]

5. Kim, K.B. Extracting Ganglion Cysts from Ultrasound Image with Fuzzy Membership Function. J. Korea Inst. Inf. Commun. Eng. 2015, 19, 1296-1300. [CrossRef]

6. Kwon, S.H.; Ryu, K.N.; Park, Y.K.; Jeong, Y.M. Soft tissue masses: Ultrasonographic findings. J. Korean Soc. Med. Ultrasound 2001, 20, 349-355.

7. Suen, M.; Fung, B.; Lung, C.P. Treatment of Ganglion Cysts. ISRN Orthop. 2013, 2013, 940615. [CrossRef]

8. Head, L.; Gencarelli, J.R.; Allen, M.; Boyd, K.U. Wrist ganglion treatment: Systematic review and meta-analysis. J. Hand Surg. 2015, 40, 546-553. [CrossRef]

9. Wang, G.; Jacobson, J.A.; Feng, F.Y.; Girish, G.; Caoili, E.M.; Brandon, C. Sonography of Wrist Ganglion Cysts: Variable and Noncystic Appearances. J. Ultrasound Med. 2007, 26, 1323-1328. [CrossRef] [PubMed]

10. Kuliński, S.; Gutkowska, O.; Mizia, S.; Martynkiewicz, J.; Gosk, J. Dorsal and volar wrist ganglions: The results of surgical treatment. Adv. Clin. Exp. Med. 2019, 28, 95-102. [CrossRef]

11. Gress, F.; Schmitt, C.; Savides, T.; Faigel, D.O.; Catalano, M.; Wassef, W.; Roubein, L.; Nickle, N.; Ciaccia, D.; Bhutani, M.; et al. Interobserver agreement for EUS in the evaluation and diagnosis of submucosal masses. Gastrointest. Endosc. 2001, 53, 71-76. [CrossRef] [PubMed]

12. Enikov, E.T.; Anton, R. Image segmentation and analysis of flexion-extension radiographs of cervical spines. J. Med. Eng. 2014, 2014, 976323. [CrossRef] [PubMed]

13. Gupta, R.; Elamvazuthi, I.; Dass, S.C.; Faye, I.; Vasant, P.; George, J.; Izza, F. Curvelet based automatic segmentation of supraspinatus tendon from ultrasound image: A focused assistive diagnostic method. Biomed. Eng. Online 2014, $13,157$. [CrossRef]

14. Suryadibrata, A.; Kim, K.B. Ganglion Cyst Region Extraction from Ultrasound Images Using Possibilistic C-Means Clustering Method. J. Inf. Commun. Converg. Eng. 2017, 15, 49-52.

15. Suryadibrata, A.; Song, D.H.; Kim, K.B. Automatic Ganglion Cyst Detection from Ultrasound Images using Fuzzy C-Means Clustering Method. Information 2017, 20, 2543-2548.

16. Bezdek, J.C.; Keller, J.; Krisnapuram, R.; Pal, N. Fuzzy Models and Algorithms for Pattern Recognition and Image Processing; Springer Science \& Business Media: New York, NY, USA, 1999.

17. Park, J.; Song, D.H.; Nho, H.; Choi, H.; Kim, K.A.; Park, H.J.; Kim, K.B. Automatic segmentation of brachial artery based on fuzzy C-means pixel clustering from ultrasound images. Int. J. Electr. Comput. Eng. 2018, 8, 638-643. [CrossRef]

18. Pei, H.X.; Zheng, Z.R.; Wang, C.; Li, C.N.; Shao, Y.H. D-FCM: Density based fuzzy c-means clustering algorithm with application in medical image segmentation. Procedia Comput. Sci. 2017, 122, 407-414. [CrossRef]

19. Huang, H.; Meng, F.; Zhou, S.; Jiang, F.; Manogaran, G. Brain image segmentation based on FCM clustering algorithm and rough set. IEEE Access 2019, 7, 12386-12396. [CrossRef]

20. Kim, K.B.; Song, D.H. Intelligent automatic extraction of canine cataract object with dynamic controlled fuzzy C-means based quantization. Int. J. Electr. Comput. Eng. 2018, 8, 666-672. [CrossRef]

21. Rehman, S.N.; Hussain, M.A. Fuzzy C-means algorithm-based satellite image segmentation. Indones. J. Electr. Eng. Comput. Sci. 2018, 9, 332-334. [CrossRef]

22. Zhou, J.; Wang, J.; Bu, H. Fabric defect detection using a hybrid and complementary fractal feature vector and FCM-based novelty detector. Fibres Text. East. Eur. 2017, 25, 46-52. [CrossRef]

23. Mohammed, K.M.C.; Kumar, S.S.; Prasad, G. Optimized Fuzzy C-means Clustering Methods for Defect Detection on Leather Surface. J. Sci. Ind. Res. 2020, 79, 833-836.

24. Park, J.; Song, D.H.; Han, S.S.; Lee, S.J.; Kim, K.B. Automatic Extraction of Soft Tissue Tumor from Ultrasonography Using ART2 Based Intelligent Image Analysis. Curr. Med. Imaging Rev. 2017, 13, 447-453. [CrossRef]

25. Kaur, J.; Choudhary, A. Comparison of Several Contrast Stretching Techniques on Acute Leukemia Images. Int. J. Eng. Innov. Technol. 2012, 2, 332-335.

26. Al-amri, S.S.; Kalyankarm, N.V.; Khamitkar, S.D. Linear and non-linear contrast enhancement image. Int. J. Comput. Sci. Netw. Secur. 2010, 10, 139-143.

27. Woo, H.S.; Kim, K.B. Improved Fuzzy Binarization Method with Trapezoid type Membership Function and Adaptive $\alpha \_c u t . J$. Korea Inst. Inf. Commun. Eng. 2016, 20, 1852-1859. [CrossRef]

28. Kanth, A.R.; Reddy, Y.N. Cubic spline for a class of singular two-point boundary value problems. Appl. Math. Comput. 2005, 170, 733-740.

29. Kim, K.B.; Lee, H.J.; Song, D.H.; Woo, Y.W. Extracting fascia and analysis of muscles from ultrasound images with FCM-based quantization technology. Neural Netw. World 2010, 20, 405-416.

30. Kim, K.B.; Park, H.J.; Song, D.H. Developing Intelligent Health Diagnosis System for Korean Traditional Medicine: Database Construction and Neural Inference. Int. J. Bio-Sci. Bio-Technol. 2014, 6, 1-8. [CrossRef] 